\title{
Fatores determinantes no tempo de tela de crianças na primeira infância
}

\author{
Determining factors in children's screen time in early childhood
}

Juliana Nogueira Pontes Nobre (https://orcid.org/0000-0002-9876-1136) ${ }^{1}$
Juliana Nunes Santos (https://orcid.org/0000-0002-1101-5270) ${ }^{2}$
Lívia Rodrigues Santos (https://orcid.org/0000-0003-3318-1592)
Sabrina da Conceição Guedes (https://orcid.org/0000-0002-9738-2302) ${ }^{2}$
Leiziane Pereira (https://orcid.org/0000-0002-8290-960X) ${ }^{2}$
Josiane Martins Costa (https://orcid.org/0000-0002-4266-4070) ${ }^{2}$
Rosane Luzia de Souza Morais (https://orcid.org/0000-0002-8236-4531) ${ }^{1,2}$
${ }^{1}$ Programa de Pós-

Graduação Saúde,

Sociedade \& Ambiente,

Universidade Federal dos

Vales do Jequitinhonha e

Mucuri (UFVJM). Rodovia

MGT 367 Km 583, 5000,

Alto da Jacuba. 39100-000

Diamantina MG Brasil.

junobre2007@yahoo.com.br

${ }^{2}$ Departamento de

Fisioterapia, UFVJM.

Diamantina MG Brasil.

${ }^{3}$ Programa de Pós-

Graduação em Ciências

Fonoaudiólogas,

Universidade Federal de

Minas Gerais (UFMG). Belo

Horizonte MG Brasil.

\begin{abstract}
The use of media by children in early childhood is increasingly common, and it is necessary to investigate the determinants of screen time, which is understood as the total child screen exposure time, including television and interactive media. This is a descriptive, exploratory, and cross-sectional study conducted with 180 children between 24 and 42 months of age, allocated in Group 1, less than two hours daily screen exposure time; Group 2, daily screen exposure time equal to or more than two hours. Bivariate and binary logistic regression analyses were performed. Screen time determining factors studied were family environment, evaluated with the Family Environment Resource Inventory; socioeconomic factors; nutritional status and child development status, evaluated with the Bayley III test. As a result, $63 \%$ of children had daily screen time exceeding two hours, and television still is the main culprit for children screen exposure. We observed that screen time exposure was positively associated with family resources, economic level, and language development. However, only the last two factors explained the longer screen time.

Key words Audiovisual media, Mobile applications, Television, Exposure time, Child development
\end{abstract}

Resumo $O$ uso de mídias por crianças na primeira infância está cada vez mais habitual, o que torna necessário investigar os fatores determinantes para o tempo de tela, entendido como o tempo total pelo qual a criança permanece exposta a todas as telas, incluindo televisão e mídias interativas. Trata-se de um estudo transversal, descritivo e exploratório, realizado com 180 crianças, entre 24 a 42 meses de idade, alocadas em: Grupo 1, exposição à tela inferior a duas horas/dia; Grupo 2, exposição à tela igual/superior a duas horas/dia. Realizou-se análise bivariada e de regressão logística binária. Os fatores determinantes no tempo de tela estudados foram os recursos do ambiente familiar, investigado por meio do Inventário de Recursos do Ambiente Familiar; fatores socioeconômicos; estado nutricional e status do desenvolvimento infantil, mensurado pelo teste Bayley III. Como resultado verificou-se que $63 \%$ das crianças apresentaram tempo de tela superior a 2 horas/dia e que a televisão ainda é a principal responsável pela exposição das crianças às telas. Observou-se que o tempo de exposição à tela esteve positivamente associado aos recursos familiares, nivel econômico e desenvolvimento da linguagem. Entretanto, apenas os dois últimos fatores explicaramo maior tempo de tela.

Palavras-chave Mídia audiovisual, Aplicativos móveis, Televisão, Tempo de exposição, Desenvolvimento infantil 


\section{Introdução}

No contexto do mundo moderno, as telas, antes restritas à televisão $0^{1,2}$, evoluíram para dispositivos de bolso, móveis e portáteis. Logo, celulares, tablets, smartphones, devido às suas portabilidades, foram incorporados na rotina de pessoas de diferentes conjunturas sociais e faixas de idade, inclusive crianças $^{3-7}$.

A infância caracteriza-se por modificações biológicas e psicossociais, que permitem aquisições importantes nos domínios motor, afetivosocial e cognitivo do desenvolvimento ${ }^{8}$. Nesse momento, o sistema nervoso central (SNC) vivência constante transformação, mielinização e organização sináptica, cujo ápice é atingido aos 24 meses, favorecendo a aprendizagem. Desta forma, o ambiente exerce grande influência por inter-relacionar de maneira constante e dinâmica com os fatores intrínsecos à criança ${ }^{8,9}$. Portanto, principalmente na primeira infância, período de 0 a 6 anos de idade segundo os documentos brasileiros $^{10}$, se deve oportunizar a criança, vínculos afetivos saudáveis, espaço adequado para a liberdade de movimento, brincadeiras livres e disponibilidade de brinquedos e/ou materiais de aprendizagem, entre outros fatores ${ }^{8,9}$.

O tempo de tela, que é entendido como o tempo total pelo qual a criança permanece exposta a todas as telas, tem aumentado ${ }^{2,11}$. Estudos indicam que a média de exposição é superior ao tempo recomendado, uma vez que a American Academy of Pediatrics (AAP) ${ }^{12}$ recomenda que o tempo de exposição de crianças à tela não ultrapasse 2 horas por dia, com conteúdos educativos e adequados para a faixa etária. Viver em um ambiente multitela, por exemplo, televisão, tablet, computadores, smartphones, pode incentivar o uso da tela precocemente na primeira infância ${ }^{13,14}$.

O tempo de exposição à tela é considerado um fator de risco para o comportamento sedentário, para doenças cardiovasculares e metabólicas em adultos. Já em crianças pode causar obesidade, maior pressão arterial e problemas relacionados à saúde mental ${ }^{15,16}$ além de reduzir o tempo de interação social e familiar ${ }^{5}$ e favorecer exposição a conteúdos impróprios ${ }^{3}$. Alguns autores associam a alta exposição à tela a atrasos nos domínios de linguagem ${ }^{1,17} \mathrm{e}$ habilidade motora fina ${ }^{18}$.

No entanto, o tempo de tela na televisão é amplamente abordado na literatura ${ }^{17,19,20}$ enquanto estudos que dizem respeito as mídias interativas (p. ex., smartphones, tablets, videogames) começaram emergir ${ }^{5,21-23}$, consoante com o aumento generalizado do acesso e uso dos dispo- sitivos móveis por crianças ${ }^{24}$. Mídias interativas podem ser definidas pelos meios tecnológicos que respondem com conteúdo às ações dos usuários, oportunizando diálogo e participação, o que diferencia da televisão $0^{25}$.

Desta forma, considerando a relevância e contemporaneidade da temática, o objetivo do presente estudo foi investigar os fatores determinantes no tempo de tela total, incluindo televisão e mídias interativas em crianças na primeira infância.

\section{Métodos}

Trata-se de um estudo transversal, descritivo e exploratório de investigação do tempo de tela em crianças de 24 a 42 meses de idade e foi realizado no período de setembro de 2016 a fevereiro de 2017. A pesquisa foi aprovada pelo Comitê de Ética em Pesquisa da Universidade Federal dos Vales do Jequitinhonha e Mucuri.

Foram incluídas no estudo crianças com idade entre 24 a 42 meses e 15 dias, regularmente matriculadas nas creches públicas e particulares da sede de um município brasileiro de pequeno porte com Índice de Desenvolvimento Humano (IDH) alto, cujos pais assinaram o Termo de Consentimento Livre e Esclarecido. Foram critérios de não inclusão crianças que apresentaram doenças congênitas ou adquiridas, que pudessem afetar o desenvolvimento cognitivo e motor ou crianças.

Para o cálculo amostral, realizado por meio do software OpenEpi; estimou-se prevalência de uso de mídias eletrônicas de $34 \%{ }^{26}$ com precisão desejada de 5\%, com intervalo de confiança de $90 \%$, poder do estudo de $80 \%$, resultando em 172 crianças após ajuste para populações finitas.

O tempo de exposição à tela diário de cada criança foi considerado para alocá-la nos grupos: Grupo 1 (G1) crianças com tempo de exposição à tela inferior a duas horas/dia; Grupo 2 (G2): crianças com tempo de exposição à tela igual ou superior a duas horas $/ \mathrm{dia}^{12}$.

Para avaliar os hábitos de mídias interativas, aplicou-se um questionário, elaborado pelos autores $^{27}$ com embasamento na literatura. O instrumento contemplou perguntas relacionadas ao conhecimento dos pais sobre as mídias interativas móveis, sua frequência de uso, tempo de exposição (em meses) as mídias pelas crianças e idade que iniciaram o acesso as mídias.

A classificação econômica foi avaliada utilizando o Critério de Classificação Econômica Brasil 2015 (CCEB), da Associação Brasileira de 
Empresas de Pesquisa. Neste, o critério, atribuise uma pontuação de acordo com os bens e grau de escolaridade do chefe da família. A partir da pontuação, classifica-se o nível econômico do grupo familiar em uma escala ordinal crescente que varia de $\mathrm{E}$ a $\mathrm{Al}^{28}$.

O desenvolvimento infantil foi mensurado utilizando-se o Bayley III que é a terceira edição da escala Bayley, utilizada em pesquisas científicas para a avaliação do desenvolvimento infantil, inclusive no Brasil ${ }^{29,30}$. O instrumento tem como objetivo identificar crianças com atraso no desenvolvimento na faixa etária de 1 a 42 meses, nos domínios: cognitivo, linguagem (receptiva e expressiva), motor (fino e grosso), socioemocional e comportamento adaptativo. Para o presente estudo, foram aplicadas as escalas cognitivas, linguagem expressiva, escala motora fina e grossa. De acordo com o manual ${ }^{31}$, cada escala é pontuada com base na somatória de tarefas realizadas pela criança, gerando escores brutos e a partir daí, escores baseados em norma para a idade. Para o presente estudo, foi utilizado o escore balanceado de cada escala, considerando que 10 é a pontuação média com desvio-padrão de \pm 3 pontos. Previamente ao estudo, a equipe realizou um treinamento para aplicação do Bayley e confiabilidade entre examinadores, obtendo um Índice de Correlação Intraclasse (ICC) de 95\%.

A qualidade do ambiente que vive a criança foi avaliada utilizando-se o Inventário de Recursos do Ambiente Familiar- (RAF) com adaptações, ou seja, foi retirada a questão 8 do instrumento, cuja intensão direcionada para crianças fora da faixa etária do estudo ${ }^{32}$. O questionário tem por objetivo avaliar os recursos do ambiente familiar em três domínios: 1) recursos que promovem processos proximais: compreende participação em experiências estimuladoras para o desenvolvimento, como passeios e viagens, oportunidades de interação com os pais; disponibilidade de brinquedos e materiais que apresentam desafio ao pensar; disponibilidade de livros, jornais e revistas, uso adequado do tempo livre; 2) atividades que sinalizam estabilidade na vida familiar: como rotinas e reuniões regulares da família e cooperação da criança em tarefas domésticas; 3 ) práticas parentais que promovem o vínculo família-escola: envolvimento direto dos pais na vida escolar, como participação nas reuniões ${ }^{33}$. Para análise foi considerado o somatório de pontos, ou seja, valores brutos.

Para avaliação do estado nutricional, dados antropométricos foram coletados por meio das medidas de peso e altura, para posterior tra- tamento e obtenção da classificação do estado nutricional da criança por meio do Anthro Who versão 3.2.2, um software desenvolvido pela Organização Mundial de Saúde ${ }^{34}$.

Os dados coletados foram transferidos para o Statistical Packege for the Social Sciences (SPSS) versão 19.0, para realização de análise exploratória do banco de dados. Para análise bivariada utilizou-se o teste Qui-quadrado, com nível de significância de 5\% $(\mathrm{p}<0,05)$. Como variável dependente foi considerado o "tempo de tela", variável constituída pela somatória dos tempos de exposição da criança às mídias eletrônicas (TV) e interativas (tablets e smartphones) por dia, segundo relato dos pais ou responsáveis. As variáveis independentes foram idade (2 e 3 anos), escolaridade materna e escolaridade paterna, nível econômico, inventário dos recursos familiares (RAF escala total e domínios separados), desenvolvimento da linguagem, desenvolvimento cognitivo, desenvolvimento motor grosso e fino, e Índice de Massa Corporal (IMC).

Todas as variáveis independentes foram categorizadas para fins de comparação entre os grupos G1 e G2. Para as variáveis que compõe o RAF utilizou-se a mediana como ponto de corte, sendo que no escore total do "Inventário de recursos familiares" considerou-se a pontuação " 50 ” para a formação das categorias abaixo e acima da mediana. Nos domínios do RAF intitulados "recursos que promovem processos proximais", "atividades que sinalizam estabilidade na vida familiar" e "práticas parentais que promovem a ligação família-creche”, os valores considerados para categorização das variáveis foram, respectivamente, 26, 16 e 8. Para o resultado do Bayley, as variáveis foram categorizadas pela pontuação 10 no escore balanceado das escalas avaliadas, por ser essa a pontuação média do teste, cujo desvio padrão é \pm 3 pontos. As variáveis escolaridade paterna e materna foram categorizadas em: até ensino fundamental completo e ensino médio ou superior. Para o nível econômico as categorias criadas foram respectivamente " $\mathrm{A}, \mathrm{B}, \mathrm{C1}$ " e " $\mathrm{C} 2$, $D, E$ ", e para o IMC obedeceu-se ao critério de magreza e eutrofia para uma categoria e indivíduos em risco de sobrepeso a obeso para outra categoria.

A associação entre as variáveis independentes e a variável dependente "tempo de tela superior a 2 horas" foi verificada na análise bivariada pelo teste Qui-quadrado com nível de 5\% de significância. Para análise multivariada utilizou-se a regressão logística binária realizada com os dados cujas variáveis independentes obtiveram p-valor 
$\leq 0,20$ na análise univariada e permaneceram no modelo apenas aquelas com $\mathrm{p}<0,05$.

A qualidade do modelo foi avaliada pelo método Hosmer-Lemeshow e pela medida de ajuste -2 log likelihood (-2LL). A análise de resíduos no modelo final foi realizada para detecção de outliers significativos.

\section{Resultados}

A Figura 1 apresenta o fluxo dos participantes do estudo, no qual participaram 180 crianças, sendo 84 do sexo masculino $(46,7 \%)$ e 96 do sexo feminino $(53,3 \%)$. A idade média das crianças foi de 35,0 $( \pm 4,5)$ meses. Das crianças avaliadas, $36(20 \%)$ estudavam em instituições privadas e 144 (80\%) em instituições públicas; 48 (26,8\%) pertencem aos estratos A e B da classificação econômica, 90 (50\%) aos estrados C e 42 (23,3\%) as classes D e E.

Quanto à escolaridade materna, 26,3\% possuem até oitava série, $45 \%$ o ensino médio completo ou superior incompleto e $28,7 \%$ possuem curso superior e/ou pós-graduação. Quanto à escolaridade paterna, 43,1\% dos entrevistados possuem até a oitava série, $36,9 \%$ ensino médio, e $20,0 \%$ possuem curso superior e/ou pós-graduação.

Da amostra de 180 participantes, 10 crianças (5,5\%) não foram expostas a nenhuma mídia. $\mathrm{Na}$ Figura 2 é possível visualizar a distribuição das

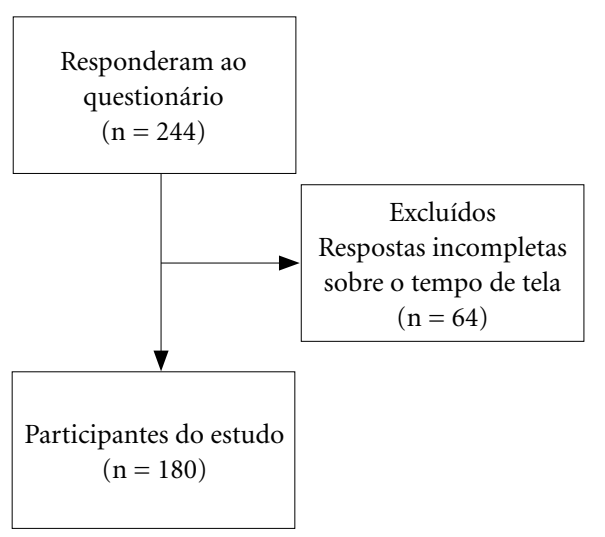

Figura 1. Fluxograma com a descrição da composição da amostra. Diamantina, Minas Gerais, Brasil, 2019.

n: número absoluto de crianças crianças quanto ao tempo de tela diário a que são expostas. $63,3 \%$ das crianças são expostas a um tempo igual ou superior a duas horas/dia.

Na Tabela 1 estão descritas as mídias interativas utilizadas pelas crianças, indicando que a mais utilizada foi a TV, seguida de smartphone e tablet. Constatou-se que poucas crianças nesta faixa fazem uso de videogames ( $4 \%$ da amostra).

Os resultados da associação entre tempo de tela e as variáveis independentes estão apresentados na Tabela 2.

Observa-se na Tabela 2 que os grupos G1 e G2 foram similares quanto à distribuição por sexo e idade, escolaridade materna e paterna, inventário dos recursos familiares, práticas parentais que promovam ligação família-creche, desenvolvimento cognitivo, motor grosso/fino e IMC. No entanto, são diferentes quanto às demais variáveis estudadas. Desta forma é possível afirmar que o nível econômico e os recursos do RAF que promovem processos proximais e o desenvolvimento da linguagem apresentaram associação estatisticamente significativa com o maior tempo de tela.

Ao ser realizada a regressão logística binária, foi feita análise de resíduos e dois outliers foram excluídos. Além disso, as interações entre as variáveis do modelo final foram examinadas e retiradas "escolaridade materna" e "inventário de recursos do ambiente familiar- RAF total”. Na Tabela 3 são apresentadas as variáveis que permaneceram no modelo de regressão logística binária.

A variável independente "nível econômico" foi considerada preditora significativa para distinção entre crianças com tempo de tela igual ou superior a duas horas e tempo de tela inferior a duas horas. Observa-se que, crianças pertencentes aos estratos superiores da classificação econômica apresentaram 3,5 vezes mais chances de terem maior exposição às telas.

A variável independente "desenvolvimento da linguagem" também foi considerada preditora significativa para distinção entre crianças com tempo de tela igual ou superior a duas horas. Observa-se que crianças com melhor desempenho na linguagem expressiva apresentaram 3,57 vezes mais chances de terem maior exposição às telas.

\section{Discussão}

Este estudo investigou os fatores determinantes para o tempo de tela de crianças de 24 a 42 meses, considerando ser este um período importante para o desenvolvimento infantil. $\mathrm{Na}$ pesquisa, 


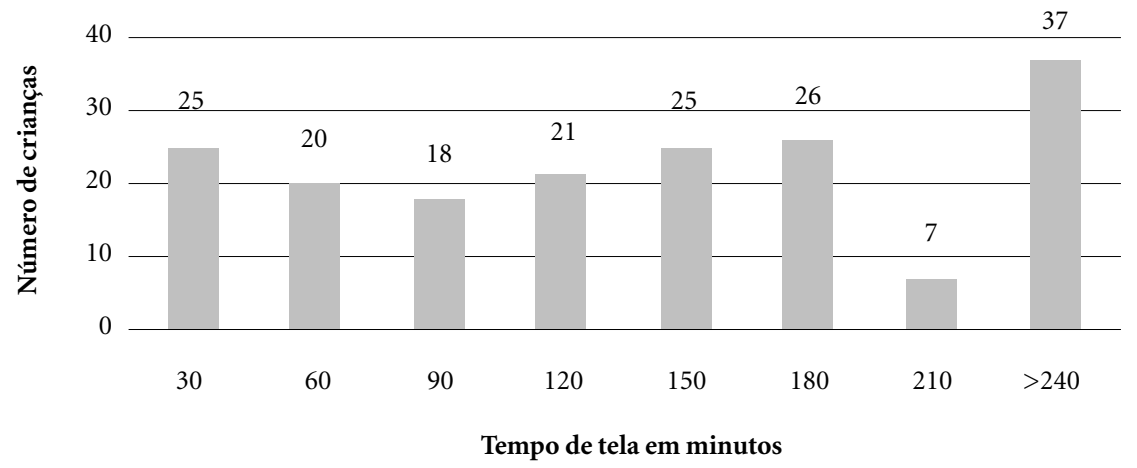

Figura 2. Distribuição do tempo de tela em minutos segundo exposição à televisão ou mídias interativas. Diamantina, Minas Gerais, Brasil, 2019.

Tabela 1. Tempo de tela especificado por tipo de mídia ( $\mathrm{n}=180)$. Diamantina, Minas Gerais, Brasil, 2019.

\begin{tabular}{lcccccc}
\hline \multirow{2}{*}{ Mídias } & \multicolumn{2}{c}{$\begin{array}{c}\text { Não } \\
\text { utilizam }\end{array}$} & \multicolumn{2}{c}{$\begin{array}{c}\text { Utilizam } \\
\text { até 2 } \\
\text { horas/dia }\end{array}$} & \multicolumn{2}{c}{$\begin{array}{c}\text { Utilizam } \\
\text { acima de 2 } \\
\text { horas/dia }\end{array}$} \\
\cline { 2 - 8 } & \multicolumn{1}{c}{$\mathbf{n}$} & \multicolumn{1}{c}{ \% } & \% & n & \% \\
\hline Smartphone & 76 & 38,8 & 34 & 20,0 & 70 & 41,2 \\
Tablet & 147 & 80,6 & 4 & 2,3 & 39 & 23,0 \\
Videogame & 171 & 94,7 & 2 & 1,2 & 7 & 4,1 \\
TV & 16 & 3,5 & 50 & 29,4 & 114 & 67,0 \\
\hline
\end{tabular}

n: número absoluto de crianças; \%: porcentagem de criança.

$63,3 \%$ das crianças tem tempo de tela superior a 2 horas/dia, concordando com os relatórios da Commom Sense Media ${ }^{35}$, Read Aloud Survey Report $^{36}$, desenvolvidos nos Estado Unidos, que também encontraram valor superior a duas horas/ dia de tempo de exposição de crianças. Estudos desenvolvidos em Cingapura ${ }^{2,11}$ também encontraram uma prevalência de mais de 2 horas/dia de exposição em crianças na primeira infância.

Os resultados das pesquisas supracitadas e também os evidenciados do presente estudo contrariam a recomendação da $\mathrm{AAP}^{12}$, que preconiza a exposição diária em até duas horas para crianças de 2 a 5 anos de idade, incluindo o uso de todas as mídias. No entanto, em um novo documento de orientações para pais e pediatras ${ }^{37}$ a recomendação da AAP se tornou mais conservadora, ou seja, o tempo de tela passou a ser de até
1 hora/dia para crianças de 2 a 5 anos de idade. Esta última recomendação também é preconizada pela Associação Brasileira de Pediatria ${ }^{38}$, entretanto, a literatura aponta estudos que afirmam que os pais têm dificuldade de incorporar a recomendação de limitação de tempo de tela na rotina de seus filhos, uma vez que o tempo de tela dos adultos também é alto ${ }^{39,40}$.

No presente estudo, $94,5 \%$ das crianças estavam expostas às telas, principalmente à televisão (61\%), seguida das mídias interativas portáteis, smartphone $(41 \%)$ e tablet $(22 \%)$. Constatou-se que poucas crianças nesta faixa de idade fazem uso de videogames (4\% da amostra), mídia popular entre crianças maiores ${ }^{35}$. Quanto à TV, a literatura associa a sua exposição intensa ao atraso da linguagem, à dificuldade de interação social, à formação do estilo de vida sedentário e pouco estímulo à criatividade $\mathrm{e}^{1,16,17,20,41}$. Embora a televisão ainda continue a ser a mídia mais comum entre crianças, é relevante considerar a tendência e o papel cada vez mais marcante de mídias interativas móveis ${ }^{2,11,42}$. Por ser considerada uma tecnologia recente, os estudos sobre as mídias interativas e seu impacto na saúde e o desenvolvimento infantil começam a emergir na literatura ${ }^{5}$. Embora ainda controverso, os estudos indicam que o uso parcimonioso destas mídias, diferente da televisão por permitir o toque de tela e interação da criança com seu conteúdo, pode contribuir positivamente para o desenvolvimento cognitivo, linguístico e motor fino infantil ${ }^{5,27,37,43-45}$.

As variáveis independentes associadas ao tempo de exposição à tela na análise bivariada 
Tabela 2. Tempo de tela e fatores associados. Diamantina, Minas Gerais, Brasil, 2019.

\begin{tabular}{|c|c|c|c|c|c|c|c|}
\hline & & & & mpod & & & \\
\hline Variá & ível & $\begin{array}{r}\text { Infer } \\
\text { hor: }\end{array}$ & $\begin{array}{l}\text { a duas } \\
n=66)\end{array}$ & $\begin{array}{r}\text { Duas } \\
\text { mais }\end{array}$ & $\begin{array}{l}\text { ras ou } \\
=114)\end{array}$ & Teste $\mathrm{X}^{2}$ & $\mathbf{p}$ \\
\hline & & $\mathbf{n}$ & $\%$ & $\mathbf{n}$ & $\%$ & & \\
\hline Sexo & Feminino & 35 & 47,0 & 61 & 53,5 & 0,04 & 0,95 \\
\hline & Masculino & 31 & 53,0 & 53 & 46,5 & & \\
\hline Idade & 2 anos & 35 & 53,0 & 55 & 48,2 & 0,20 & 0,53 \\
\hline & 3 anos & 31 & 47,0 & 59 & 51,8 & & \\
\hline Escolaridade materna & $\begin{array}{l}\text { Até Ens. Fundamental } \\
\text { completo }\end{array}$ & 21 & 34,4 & 24 & 21,8 & 3,21 & 0,07 \\
\hline & Ens. Médio ou superior & 40 & 65,6 & 86 & 78,2 & & \\
\hline Escolaridade paterna & $\begin{array}{l}\text { Até Ens. Fundamental } \\
\text { completo }\end{array}$ & 32 & 52,5 & 37 & 37,4 & 3,50 & 0,06 \\
\hline & Ens. Médio ou superior & 29 & 47,5 & 62 & 62,6 & & \\
\hline Nível socioeconômico & $\mathrm{A}, \mathrm{B}$ e $\mathrm{C} 1$ & 23 & 34,8 & 65 & 58,0 & 8,93 & $<0,01^{\star}$ \\
\hline & $\mathrm{C} 2, \mathrm{D}$ e $\mathrm{E}$ & 43 & 65,2 & 47 & 42,0 & & \\
\hline Inventário dos recursos & Pontuação até 50 & 39 & 59,1 & 54 & 47,4 & 2,30 & 0,12 \\
\hline familiares (RAF)- total & Pontuação acima de 50 & 27 & 40,9 & 60 & 52,6 & & \\
\hline Recursos que promovem & Pontuação até 26 & 38 & 65,5 & 47 & 44,8 & 6,45 & $0,01^{*}$ \\
\hline processos proximais (RAF) & Pontuação acima de 26 & 20 & 34,5 & 58 & 55,2 & & \\
\hline Atividades que sinalizam & Pontuação até 16 & 28 & 48,3 & 54 & 51,4 & 0,14 & 0,70 \\
\hline $\begin{array}{l}\text { estabilidade na vida familiar } \\
\text { (RAF) }\end{array}$ & Pontuação acima de 16 & 30 & 51,7 & 51 & 48,6 & & \\
\hline Práticas parentais que & Pontuação até 8 & 47 & 71,2 & 81 & 71,1 & 0,01 & 0,98 \\
\hline $\begin{array}{l}\text { promovem a ligação } \\
\text { família-creche (RAF) }\end{array}$ & Pontuação acima de 8 & 19 & 28,8 & 33 & 28,9 & & \\
\hline Desenvolvimento da & Pontuação até 9,9 & 16 & 45,7 & 14 & 22,6 & 5,60 & $0,01^{\star}$ \\
\hline Linguagem & Pontuação acima de 10 & 19 & 54,3 & 48 & 77,4 & & \\
\hline Desenvolvimento Cognitivo & Pontuação até 9,9 & 18 & 48,6 & 29 & 43,3 & 0,27 & 0,59 \\
\hline & Pontuação acima de 10 & 19 & 51,4 & 38 & 56,7 & & \\
\hline Desenvolvimento Motor & Pontuação até 9,9 & 42 & 77,8 & 63 & 69,2 & 1,23 & 0,26 \\
\hline Grosso & Pontuação acima de 10 & 12 & 22,2 & 28 & 30,8 & & \\
\hline Desenvolvimento Motor & Pontuação até 9,9 & 12 & 27,9 & 26 & 33,3 & 0,37 & 0,58 \\
\hline Fino & Pontuação acima de 10 & 31 & 72,1 & 52 & 66,7 & & \\
\hline IMC & Magreza e eutrofia & 37 & 66,1 & 66 & 71,7 & 0,52 & 0,46 \\
\hline & $\begin{array}{l}\text { Risco de sobrepeso, } \\
\text { sobrepeso e obesidade }\end{array}$ & 19 & 33,9 & 26 & 28,3 & & \\
\hline
\end{tabular}

Tabela 3. Regressão logística binária hierárquica para variável dependente “Tempo de tela”. Diamantina, Minas Gerais, Brasil, 2019.

\begin{tabular}{lcccc}
\hline \multirow{2}{*}{ Variáveis independentes } & \multicolumn{4}{c}{ Tempo de tela } \\
\cline { 2 - 5 } & b \pm S.E & OR & IC $(\mathbf{9 5 \% )}$ & $\mathbf{p}$ \\
\hline Nível econômico & $1,26 \pm 0,59$ & 3,538 & $1,11-11,25$ & $0,03^{*}$ \\
Desenvolvimento da linguagem & $1,27 \pm 0,59$ & 3,570 & $1,12-11,34$ & $0,03^{*}$ \\
Recursos que promovem processos proximais & & & & 0,13 \\
Escolaridade paterna & & & & 0,75 \\
\hline
\end{tabular}

Qualidade do modelo segundo Hosmer e Lemeshow $=0,92 .{ }^{*} \mathrm{p}=$ nível de significância $<0,05$. b=coeficiente de regressão.

S.E=Margem de Erro. OR=Razão de Chance, IC=Intervalo de confiança. 
foram: nível econômico, recursos no ambiente familiar que desenvolvem processos proximais $\mathrm{e}$ desenvolvimento da linguagem, A escolaridade paterna e materna, embora não tenham apresentado a significância estatística de $95 \%$, obtiveram valores de $p<0,20$, e seguiram para a etapa de regressão logística.

A escolaridade paterna está associada a melhores oportunidades de estimulação no $\operatorname{lar}^{46}$. Esta estimulação pode ser entendida, como por exemplo, por oferecer um ambiente que contenha recursos para aprendizagem, no qual as mídias interativas também estejam presentes. Sugere-se ainda que os pais que tenham maior escolaridade façam mais o uso de mídia em sua rotina o que pode influenciar diretamente no tempo de tela da criançç $^{39,40}$.

A escolaridade materna tem sido apontada como uma importante preditora para o crescimento, a saúde e o desenvolvimento infantili ${ }^{47,48}$. É possível que as mães com maior escolaridade materna disponibilizem maiores recursos para o desenvolvimento infantil, utilizando a mídia interativa como recurso para promoção do aprendizado. Vectore et al. ${ }^{49}$ fazem uma reflexão sobre a criança na contemporaneidade e afirmam que a vida em sociedades altamente competitivas intensificou a preocupação e o desejo da mãe moderna na busca de super qualificação dos filhos, com atividades diversificadas e exposição precoce ao mundo do adulto altamente tecnológico, visando um futuro distante de sucesso na fase adulta, mas construído desde os primeiros anos de vida.

Os recursos que promovem processos proximais apresentaram associação positiva com o maior tempo de tela. Quanto mais disponibilidade de brinquedos e materiais que aprendizagem em casa, maior a possibilidade de haver recursos tecnológicos como mídias interativas, computadores e televisão e, portanto, maior tempo de exposição às telas ${ }^{35,37}$.

$\mathrm{Na}$ presente pesquisa, as variáveis independentes que se associaram ao tempo de tela e que permaneceram na análise multivariada foram respectivamente, nível econômico e linguagem, consideradas preditoras significativas para distinção entre os grupos com tempo de tela igual ou superior às $2 \mathrm{~h}$ e tempo de tela inferior às $2 \mathrm{~h}$. Cada qual aumenta, respectivamente, em 3,5 e 3,57 as chances do tempo de tela maior.

Quanto mais elevado o nível econômico, maior a possibilidade de aquisição de diferentes mídias, consequentemente, maior tempo de tela $^{35,37}$. Embora a TV tenha se popularizado, com preços mais acessíveis, os smartphones e, principalmente, o tablet são de valor aquisitivo mais elevado. Estudos afirmam que nos Estados Unidos $^{35,37}$, a disponibilidade de smartphones, e particularmente de tablets, é menor em famílias de baixa renda. Percebe-se que o Brasil segue esta tendência.

Sobre isso, Simões et al. ${ }^{50}$, em pesquisa realizada em Portugal, afirmam que a posse do tablet é mais evidente em lares de estratos socioeconômicos médios em relação aos estratos mais baixos no continente europeu, portanto, as classes mais elevadas possuem além do tablet, computadores portáteis, dentre outras mídias, enquanto aqueles cujos meios socioeconômicos são mais baixos possuem menos equipamentos para seu uso individual. A literatura afirma que a divisão digital entre níveis econômicos é aparente no conteúdo do programa dos dispositivos móveis e, segundo a Comon Sense Media ${ }^{35}$, as famílias de renda mais alta instalam mais aplicativos especificamente para seus filhos, incluindo jogos e aplicativos educacionais.

Quanto à linguagem, há associação entre o uso da TV a atrasos na linguagem em crianças $^{1,17,19,20,41}$. Entretanto, o presente estudo investigou o tempo de tela, incluindo as mídias interativas. Reich et al. ${ }^{51}$, em uma revisão de pesquisas em aprendizagem de crianças a partir de telas afirmam que, ebooks bem projetados propiciam às crianças aprendizagem igualmente bem, $e$ às vezes mais do que dos livros impressos. Porém, os autores ressaltam que ebooks aprimorados com sons, animações e jogos podem distrair as crianças e reduzir a aprendizagem.

Há evidências na literatura de que aplicativos educacionais contribuem para o aumento lexical em crianças ${ }^{26}$ e pode ensinar habilidades de leitura e alfabetização ${ }^{7,43}$. Consoante com este entendimento, em seu estudo sobre interatividade, toque de tela e aprendizagem da criança, Russo-Johnson et al. ${ }^{44}$ associaram positivamente o uso de tablet na aprendizagem de palavras com provável transferência de aprendizagem ao objeto real, corroborando com Huber et al. ${ }^{52}$, que identificaram em sua pesquisa com crianças de 4 a 6 anos de idade, a capacidade de aprender e transferir aprendizagem de um dispositivo de toque de tela, aplicando esse conhecimento em suas interações.

No entanto, a literatura ${ }^{5,12,27,37,38}$ enfatiza a importância de se levar em consideração alguns fatores para o uso das mídias interativas por crianças na primeira infância: a restrição do tempo e seu conteúdo; atividades interativas versus atividades passivas; uso para diversão ou apren- 
dizado em contraposição ao uso para "deixar a criança quieta" e principalmente, a importância da presença do adulto como mediador. A presença de um adulto compartilhando com a criança a experiência de leitura para interpretar, dialogar e discutir propicia uma melhor interpretação e estimula o desenvolvimento da linguagem, o que diferencia do fato da criança interagir por ela mesma com a mídia interativa ${ }^{5,27,51,53}$.

No Brasil, os estudos sobre tempo de tela e fatores associados ainda são incipientes, mas é notória a existência de uma demanda atual no cuidado da população pediátrica, no que se refere à construção de parâmetros para o uso das telas por crianças na primeira infância, visto que há influência do tempo, forma e qualidade da exposição às telas no desenvolvimento ${ }^{12,27,38}$. Dessa maneira, o presente estudo, de forma pioneira, buscou compreender os fatores associados ao tempo de tela em uma amostra representativa de crianças brasileiras a fim de se contribuir para a construção de evidências para aplicabilidade em intervenções futuras benéficas ao desenvolvimento infantil.

Como limitações da pesquisa aponta-se o emprego de questionário preenchido pelos pais, o que favorece o viés de memória e desejabilidade social, entretanto esta ainda é a forma mais utilizada em outras pesquisas ${ }^{2,11,19,21,23}$. Sugere-se como perspectivas futuras, investigar o tempo de tela dos pais, no intuito de verificar se há associação com a utilização de mídia por parte das crianças $^{3,39,40}$, visto a necessidade de incrementar estudos dessa natureza para a área de saúde coletiva, sejam eles de natureza quantitativa como o presente estudo ou qualitativa, a fim de entender em profundidade os fenômenos associados ao tempo de tela em crianças.

\section{Conclusão}

As crianças do presente estudo apresentaram tempo de tela acima do recomendado para a sua idade. A televisão ainda foi a principal responsável pela exposição das crianças às telas, contribuindo substancialmente para esse contato. Observou-se que o tempo de exposição à tela foi positivamente associado, aos recursos familiares, ao nível econômico, desenvolvimento da linguagem, no entanto, apenas as duas últimas explicaram o maior tempo de tela.

\section{Colaboradores}

JNP Nobre: coleta e interpretação dos dados; escrita e revisão crítica do manuscrito. JM Costa, LR Santos, SC Guedes e L Pereira: coleta dos dados e revisão crítica do manuscrito. JN Santos e RLS Morais: concepção e orientação do trabalho; interpretação dos dados; escrita e revisão crítica do manuscrito.

\section{Agradecimentos}

Gostaríamos de expressar nossa gratidão à Secretaria Municipal de Educação de Diamantina, aos coordenadores, aos pais e as crianças participantes. Agradecemos ao programa de Pós-Graduação Saúde, Sociedade \& Ambiente (PPGSaSA) da Universidade Federal dos Vales do Jequitinhonha e Mucuri (UFVJM). 


\section{Referências}

1. Valdivia AI, Gárate SE, Regal CN, Castillo IG, Sáez ZM. Exposición a televisión y retardo primario del lenguaje en menores de 5 años. Rev Cubana Pediatr 2014; 86(1):18-25.

2. Bernard JY, Padmapriya N, Chen B, Cai S, Tan KH, Yap F, Shek L, Chong YS, Gluckman PD, Godfrey KM, Kramer MS, Saw SM. Predictors of screen viewing time in young Singaporean children: the GUSTO cohort. IJBNPA 2017; 14(1):112.

3. Straker L, Pollock C. Optimizing the interaction of children with information and communication technologies. Ergonomics 2005; 48(5):506-521.

4. Carson V, Janssen I. Associations between factors within the home setting and screen time among children aged $0-5$ years: a cross-sectional study. BMC Public Health 2012; 12(1):539.

5. Radesky JS, Schumacher J, Zuckerman B. Mobile and Interactive Media Use by Young Children: The Good, the Bad, and the Unknown. Pediatrics 2015; 135(1):13.

6. Kirkorian HL, Pempek TA, Murphy LA, Schmidt ME, Anderson DR. The Impact of Background Television on Parent-Child Interaction. Child Dev 2009; 80(5):1350-1359.

7. Radesky JS, Dimitri A. Christakis DA. Increased Screen Time: implications for early childhood development and behavior. Pediatr Clin N Am 2016; 63:827839.

8. Black MM, Walker SP, Fernald LCH, Andersen CT, DiGirolamo AM, Lu C, McCoy DC, Fink G, Shawar YR, Shiffman J, Devercelli AE, Wodon QT, Vargas-Barón E, Grantham-McGregor S. Early childhood development coming of age: science through the life course. Lancet 2016; 389(10064):77-90.

9. Daelmans B, Darmstadt GL, Lombard J, Black MM, Britto PR, Lye S, Bhutta TDZA, Richter LM. Early childhood development: the foundation of sustainable Development. Lancet 2016; 389(10064):9-11.

10. Brasil. Ministério da Saúde (MS). Secretaria de Atenção à Saúde. Departamento de Ações Programáticas e Estratégicas em Saúde. O futuro hoje: estratégia brasileirinhas e brasileirinhos saudáveis: primeiros passos para o desenvolvimento nacional. Brasília: MS; 2010.

11. Goh SN, Teh LH, Tay WR, Anantharaman S, Van Dam RM, Tan CS. Sociodemographic, home environment and parental influences on total and device-specific screen viewing in children aged 2 years and below: an observational study. BMJ Open 2016; 6:e009113.

12. American Academy of Pediatrics. Children, Adolescents, and the Media. Pediatrics 2013; 132(5):958-961.

13. Cristakis CA. Interactive Media Use at Younger Than the Age of 2 Years Time to Rethink the American Academy of Pediatrics Guideline. JAMA Pediatr 2014; 168(5):399-400.

14. Ferrari GLM, Araújo TL, Oliveira LC, Matsudo V, Fisberg M. Association between electronic equipment in the bedroom and sedentary lifestyle, physical activity, and body mass index of children. J Pediatr 2015; 91(6):574-582

15. Bento GG, Silva FC, Gonçalves E, Santos PD, Silva R. Revisão sistemática sobre nível de atividade física e estado nutricional de crianças brasileiras. Revista Salud Pública 2016; 18(4):630-642.
16. Dutra GF, Kaufmann CC, Pretto ADB, Albernaz EP. Television viewing habits and their influence on physical activity and childhood overweight. J Pediatr 2015; 91(4):346-351.

17. Lin LY, Cherng RJ, Chen YJ, Chen YJ, Yang HM. Effects of television exposure on developmental skills among young children. Infant Behav Dev 2015; 38:20-26.

18. Lin LY, Cherng RJ, Chen YJ. Effect of Touch Screen Tablet Use on Fine Motor Development of Young Children. Phys Occup Ther Pediatr 2017; 37(5):457-467.

19. Duch H, Fisher EM, Ensari I, Font M, Harrington A, Taromino C, Yip J, Rodriguez C. Association of screen time use and language development in Hispanic toddlers: a cross-sectional and longitudinal study. Clin Pediatr (Phila) 2013; 52(9):857-865.

20. Okuma K, Tanimura M. A preliminary study on the relationship between characteristics of TV content and delayed speech development in young children. Infant Behav Dev 2009; 32(3):312-321.

21. Cristia A, Seidl A. Parental Reports on Touch Screen Use in Early Childhood. PloS One 2015; 10(6):e0128338.

22. Karaagac, AT. Undesirable Effects of Media on Children: Why Limitation is Necessary? Indian Pediatr 2015; 52(6):469-471.

23. Kabali HM, Nunez-Davis R, Budacki JM, Mohanty $\mathrm{JH}$, Leister KP, Bonner RL. Exposure and use of mobile media devices by young children. Pediatrics 2015; 136(6):1044-1050.

24. Paudel S, Leavy J, Jancey J. Correlates of mobile screen media use among children aged 0-8: protocol for a systematic review. Syst Rev 2016; 5(1):91.

25. Nicolau M. Fluxo, conexão, relacionamento: um modelo comunicacional para as mídias interativas. Culturas Midiáticas 2008; 1(1):1-10.

26. Rideout V. Learning at home: Families educational media use in America [Internet]. The Joan Ganz Cooney Center; 2014. [acessado 2018 Dez 10] Disponível em: http://www.academia.edu/6444806/The_Learning_ at_home_families_educational_media_use_in_america

27. Nobre JNP, Vinolas Prat B, Santos JN, Santos, LR, Pereira L, Ribeiro RF, Guedes SC, Morais, RLS. Qualidade de uso de mídias interativas na primeira infância e desenvolvimento infantil: uma análise multicritério. $J$ Pediatr 2020; 96(3):310-317.

28. Associações Brasileiras de Empresas de Pesquisa (ABEP). Critério de classificação econômica Brasil [Internet]. 2015 [acessado 2016 Jan 26]. Disponível em: http://www.abep.org/criterio-brasil

29. Madaschi V, Mecca TP, Macedo EC, Paula CS. Bayley -III Scales of Infant and Toddler Development: Transcultural Adaptation and Psychometric Properties. Paidéia 2016; 26(64):189-197.

30. Souza CT, Santos DC, Tolocka RE, Baltieri L, Gibim NC, Habechian FAP. Avaliação do desempenho motor global e em habilidades motoras axiais e apendiculares de lactentes frequentadores de creche. Rev Bras Fisioter 2010; 14(4):309-315.

31. Bayley N. Bayley scales of infant and toddler development: technical manual. San Antonio: Pearson; 2006. 
32. Guimarães AF, Carvalho DV, Machado NAA, Baptista $\mathrm{R}$ AN, Lemos SMA. Risk of developmental delay of children aged between two and 24 months and its association with the quality of family stimulus. Rev Paul Pediatr 2013; 31(4):452-458.

33. Marturano EM. O inventário de recursos do ambiente familiar. Psicol Reflexão Crítica 2006; 19(3):498-506.

34. WHO Anthro for Mobile Devices. Manual Software for assessing growth and development of the world's children. Genebra: WHO; 2011.

35. Common Sense Media. Zero to eight: Children's media use in America [Internet]. 2011 [acessado 2018 Jan 26] Disponível em: https://www.commonsensemedia. org/research/zero-to-eight-childrens-media-use-in -america

36. Read Aloud Survey Report. Yougov. Report: How America reads aloud to its children [Internet]. 2016[acessado 2019 Jan 04] Disponível em: http://www.readaloud.org/documents/ReadAloudSurveyReport.pdf

37. Council on Communications Media. Media and young minds. Pediatrics 2016; 138(5):e20162591.

38. Sociedade Brasileira de Pediatria (SBP). Saúde da Criança e Adolescentes na Era Digital. Manual de Orientação. Departamento de Adolescência: SBP; 2016.

39. Schoeppe S, Rebar AL, Short CE, Alley S, Lippevelde WV, Vandelanotte C. How is adults' screen time behaviour influencing their views on screen time restrictions for children? A cross-sectional study. BMC Public Health 2016; 16:201.

40. Assathiany R, Guery E, Caron FM, Cheymol J, Picherot G, Foucaud P, Gelbert N, Association française de pédiatrie ambulatoire, Groupe de pédiatrie générale. Children and screens: A survey by French pediatricians. Arch Pediatr 2018; 25(2):84-88.

41. Chonchaiya W, Pruksananonda C. Television viewing associates with delayed language development. Acta Paediatrica 2008; 97(7):977-982.

42. Solomon-Moore E, Sebire SJ, Macdonald-Wallis C, Thompson J, Lawlor D, Jago R. Exploring parents' screen-viewing behaviours and sedentary time in association with their attitudes toward their young child's screen-viewing. Prev Med Rep 2017; 7:198-205.

43. Anderson D, Subrahmanyam K. Digital Screen Media and Cognitive Development. Pediatrics 2017; 40(2):57-62.

44. Russo-Johnson C, Troseth G, Duncan C, Mesghina A. Tapped Out: Touchscreen Interactivity and Young Children's Word Learning. Front Psychol 2017; 8:578.

45. Skaug S, Englund KT, Saksvik-Lehouillier I, Lydersen S, Wichstrøm L. Parent-child interactions during traditional and interactive media settings: A pilot randomized control study. Scand J Psychol 2018; 59:135-145.
46. Defilipo EC, Frônio JC, Teixeira MT, Leite ICB, Bastos RR, Vieira MT, Ribeiro LC. Oportunidades do ambiente domiciliar para o desenvolvimento motor. Rev Saude Publica 2012; 46(4):633-641.

47. Morais RLS, Carvalho AM, Magalhães LC. O contexto ambiental e o desenvolvimento na primeira infância: estudos brasileiros. J Phys Educ 2016; 27(1):2714.

48. Klein CJ, Madi JM, Araújo BF, Zatti H, Bosco DS, Henke CN, Rombaldi RL, Madi SRC. Fatores de risco relacionados à mortalidade fetal. Rev AMRIGS 2012; 56(1):11-16.

49. Vectore C, Rebello SMP, Vectore PT, Dias SAC. (2018). "Ele foi orçado, mas não planejado!": a infância na contemporaneidade. Rev CES Psic 2018; 11(2):37-52.

50. Simões JA, Ponte C, Ferreira E, Doretto J, Azevedo C. Crianças e Meios Digitais Móveis em Portugal: Resultados Nacionais do Projeto Net Children Go Mobile. Lisboa: CESNOVA; 2014.

51. Reich SM, Yau JC, Warschauer M. Tablet-Based eBooks for Young Children: What Does the Research Say? J Dev Behav Pediatr2016; 37(7):585-591.

52. Huber B, Tarasuik J, Antoniou M N, Garret C, Bowe SJ. Young children's transfer of learning from a touchscreen device. Comput Human Behav 2016; 56:5664.

53. Hiniker A, Sobel K, Ray Hong S, Suh H, Irish I, Kim D, Kientz JA. Touchscreen Prompts for Preschoolers: Designing Developmentally Appropriate Techniques for Teaching Young Children to Perform Gestures. Human Centered Design and Engineering University of Washington., Medford, MA, USA 2015; 21(25):109118.

Artigo apresentado em 11/09/2018

Aprovado em 10/06/2019

Versão final apresentada em 12/06/2019

Editores-chefes: Romeu Gomes, Antônio Augusto Moura da Silva 